\title{
Chylothorax and lymphedema as the initial manifestations of gastric carcinoma: A case report and review of the literature
}

\author{
JIE WU ${ }^{1}$, LIANG LV ${ }^{1}$, KUNYAN ZHOU ${ }^{2}$ and JIRONG HUO ${ }^{1}$ \\ ${ }^{1}$ Department of Gastroenterology, The Second Xiangya Hospital of Central South University, Changsha, Hunan 410011; \\ ${ }^{2}$ Department of Gastroduodenal Surgery, Hunan Cancer Hospital of Xiangya Medical School, \\ Central South University, Changsha, Hunan 410013, P.R. China
}

Received January 14, 2015; Accepted September 4, 2015

DOI: $10.3892 / \mathrm{ol} .2016 .4285$

\begin{abstract}
Chylothorax is the accumulation of lymph fluid in the pleura. Gastric carcinoma with chylothorax and lymphedema as the initial manifestations has rarely been reported, with only 14 cases reported to date. The mechanisms of gastric carcinoma generating chylothorax have not yet been determined. The current study reports the case of a 63-year-old woman presenting with chylothorax and lower extremity lymphedema, and reviews the existing literature. A chest radiograph performed on the present patient revealed large pleural effusion and chylothorax was diagnosed. Upper gastrointestinal endoscopy identified an irregular apophysis lesion and a biopsy confirmed poorly differentiated gastric adenocarcinoma. The patient was discharged at her request, and subsequently succumbed to the disease 4.5 months later. Based on the findings of the present study, as well as those of the literature, we proposed a novel form of gastric carcinoma infiltrating the body. Chylothorax and lymphedema may be a consequence of gastric carcinoma cells infiltrating the lymphatic circulatory system; therefore, the differential diagnosis of chylothorax and lymphedema of unknown cause should consider gastric carcinoma, regardless of gastrointestinal symptoms.
\end{abstract}

\section{Introduction}

Gastric cancer is a worldwide health issue that is commonly associated with gastrointestinal symptoms. Chylothorax refers to the accumulation of lymphatic fluid in the pleura, due to the obstruction or disruption of the thoracic duct $(1,2)$. Gastric

Correspondence to: Professor Jirong Huo, Department of Gastroenterology, The Second Xiangya Hospital of Central South University, 139 Renmin Middle Road, Changsha, Hunan 410011, P.R. China

E-mail: jironghuo@hotmail.com

Abbreviations: CEA, carcinoembryonic antigen; ADA, adenosine deaminase; $\mathrm{LDH}$, dehydrogenase

Key words: gastric carcinoma, chylothorax, lymphedema, pleural effusion carcinoma with chylothorax and lymphedema as the initial manifestations has rarely been reported. To date, only 14 case reports of gastric carcinoma associated with chylothorax and lymphedema are available (3-16).

When a patient presents with chylothorax, the gastric cancer is typically at an advanced stage and, thus, the prognosis is considerably poorer compared to the survival time of patients with gastric cancer without chylothorax; however, the mechanisms of gastric carcinoma-induced chylothorax have yet to be elucidated. Limited information has been provided by the existing literature; of note, in the 14 previously reported cases reviewed in the present study, chylothorax has been demonstrated to be closely associated with the presence of lymphedema. However, it is unclear how lymphedema affects the outcome of patients with chylothorax.

The current study presents the rare case of a 63-year-old woman diagnosed with gastric carcinoma, presenting with the initial manifestations of chylothorax and lymphedema of the lower extremities, and reviews the literature. The aim of the present case report is to obtain an improved understanding of the mechanisms of gastric carcinoma-induced chylothorax and lymphedema in order to achieve a earlier diagnosis of gastric cancer.

\section{Case report}

A 63-year-old woman with no relevant medical history was admitted to The Second Xiangya Hospital of Central South University (Changsha, China) in March 2014, presenting with gradual bilateral lower extremity swelling for 8 months and dyspnea for 4 months. No obvious weight loss or fever was observed, and no complaints of gastrointestinal symptoms were noted during the last 5 months. The patient only complained of decreased appetite. Physical examination showed dullness to percussion with decreased breath sounds at the right thorax, as well as mild bilateral non-pitting edema of the lower extremities. Two enlarged lymph nodes were identified upon palpation: A left supraclavicular lymph node measuring $2 \times 2.5 \mathrm{~cm}$ and a right neck lymph node measuring $1 \times 1.5 \mathrm{~cm}$.

The laboratory examinations performed revealed the following: Routine blood and urine tests, renal function tests and tuberculosis-spot tests were all normal; however, the fecal 
occult blood test was weakly positive. Tumor markers identified in the blood were as follows: Carcinoembryonic antigen (CEA), $6.830 \mathrm{ng} / \mathrm{ml}$ (normal value in blood, $<5.000 \mathrm{ng} / \mathrm{ml}$ ); and cancer antigen $125,205.82 \mathrm{kU} / 1$ (normal value, $<35.00 \mathrm{kU} / \mathrm{l}$ ).

A chest radiograph $\left(\right.$ Brivo $^{\text {TM }}$ DR-F X-ray system; GE Healthcare, Chicago, IL, USA) revealed a large pleural effusion on the right side and a small pleural effusion on the left (Fig. 1). Ultrasound confirmed the enlargement of the left supraclavicular and right neck lymph nodes with the addition of minimal ascites. No other enlarged superficial lymph nodes were detected by the ultrasound. Furthermore, a computed tomography (CT) scan revealed a clearly thickened gastric antrum wall and an enlarged retroperitoneal lymph node measuring $1.0 \times 1.2 \mathrm{~cm}$ with a small pericardial effusion, but no obvious enlarged mediastinal lymph nodes.

Following admission, turbid-yellow fluid ( $500 \mathrm{ml})$ was obtained from the space between the lungs and the chest wall by right-sided thoracentesis. The effusion contained 1,250x10 $/ 1$ leukocytes, with $29.7 \mathrm{~g} / 1$ albumin (32.4 g/l serum albumin), 136.5 U/1 lactate dehydrogenase (LDH), 7.2 U/1 adenosine deaminase (ADA), $5.80 \mathrm{mmol} / 1$ glucose and $19.04 \mathrm{ng} / \mathrm{ml} \mathrm{CEA}$ (normal value in pleural effusion, $<6.500 \mathrm{ng} / \mathrm{ml}$ ). Thoracentesis was performed again 7 days later, yielding $400 \mathrm{ml}$ milky fluid containing $4.43 \mathrm{mmol} / \mathrm{l}$ triglycerides $(1.56 \mathrm{mmol} / \mathrm{l}$ serum triglycerides), $2.92 \mathrm{mmol} / \mathrm{l}$ cholesterol $(5.13 \mathrm{mmol} / 1 \mathrm{serum}$ cholesterol), $25.1 \mathrm{~g} / 1$ albumin, 117.2 U/1 LDH, 5.8 U/1 ADA, $6.98 \mathrm{mmol} / 1$ glucose, 1,200x10 $/ 1$ leukocytes and $17.33 \mathrm{ng} / \mathrm{ml}$ CEA. The examination for chylomicrons in the pleural effusion was positive. The difference in the factors identified in the first and second effusions was due to the alteration in the diet of the patient; between the first and second effusion the patient increased her intake of dietary fat leading to an increase in triglycerides and chylomicrons and the milky appearance of the effusion fluid. An irregular apophysis lesion measuring $3.0 \times 3.5 \mathrm{~cm}$ was observed at the gastric fundus by upper gastrointestinal endoscopy (Fig. 2) (GIF-PQ260 Video Gastroscope; Olympus Corporation, Tokyo, Japan). Hematoxylin and eosin staining of the biopsy was undertaken at the Department of Pathology, The Second Xiangya Hospital of Central South University, and confirmed a poorly differentiated gastric adenocarcinoma (Fig. 3). The patient refused further treatment and follow-ups, and was discharged in April 2014 at her own request. The patient succumbed 4.5 months following the diagnosis of chylothorax in August 2014.

Written informed consent was obtained from the patient's family for the publication of the study.

\section{Discussion}

Chylothorax is diagnosed based on the presence of chylomicrons in the pleural effusion. In the majority of cases, it occurs following thoracic surgery and it is associated with malignant tumors, particularly lymphoma $(1,2)$.

To date, 20 cases of chylothorax and lymphedema associated with gastric carcinoma can be found in the literature, however, the full text or abstract could be accessed in only 14 of them (3-16). Despite the publication of these cases, the mechanism of gastric carcinoma-induced chylothorax has yet to be determined. Data collected from the present case and the 14 cases found in the literature are presented in Table I.

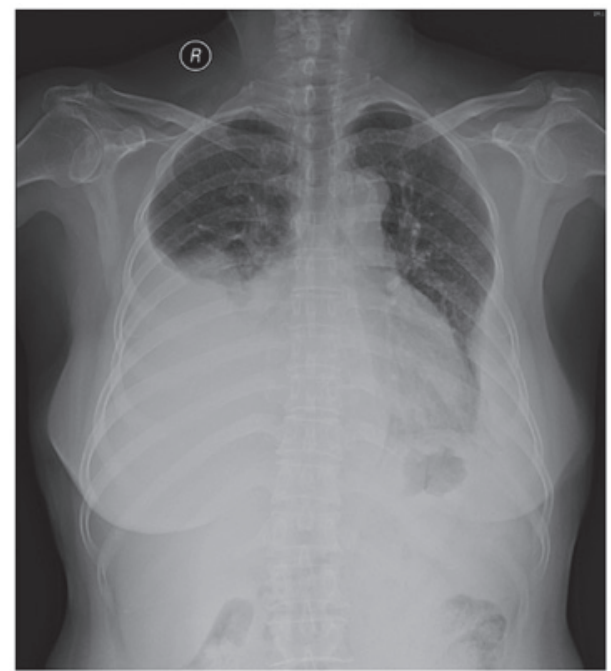

Figure 1. Chest X-ray revealing a large pleural effusion on the right (R) side and a small pleural effusion on the left.

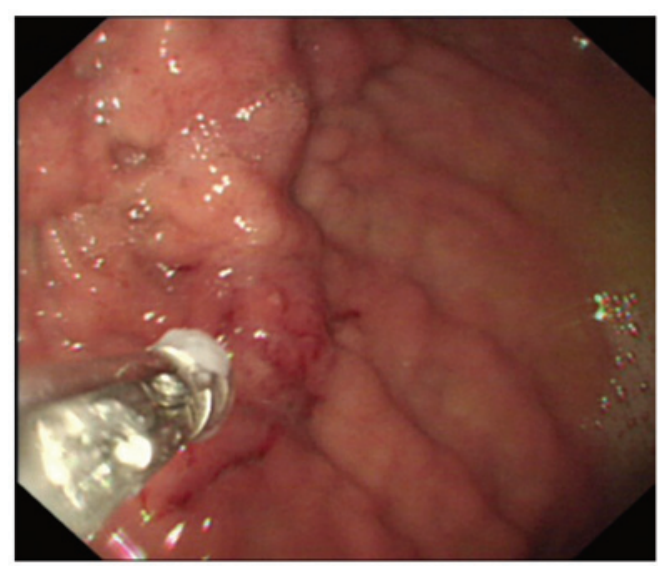

Figure 2. Gastric endoscopy showing an irregular apophysis lesion at the gastric fundus measuring $3.0 \times 3.5 \mathrm{~cm}$.

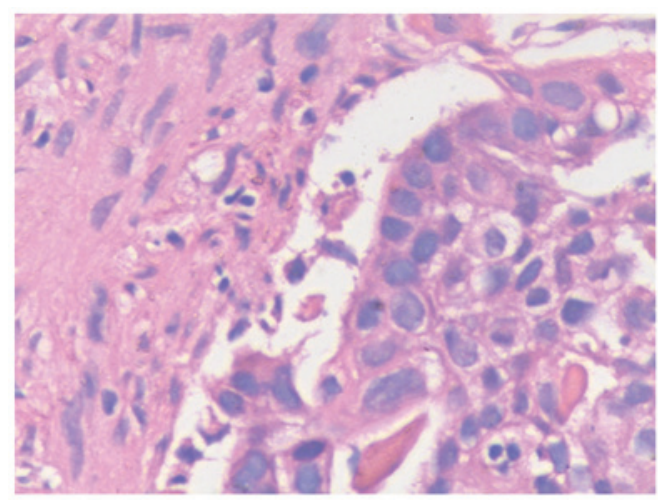

Figure 3. Biopsy of the irregular apophysis lesion at the gastric fundus indicating poorly differentiated gastric adenocarcinoma (hematoxylin and eosin staining; magnification, $\mathrm{x} 400$ ).

The patients from the reviewed case reports typically presented with respiratory rather than gastrointestinal symptoms. Of the 14 cases, 9 exhibited signet ring cell carcinoma $(4-6,8-10,12,14,15)$ and 3 had poorly differentiated gastric 


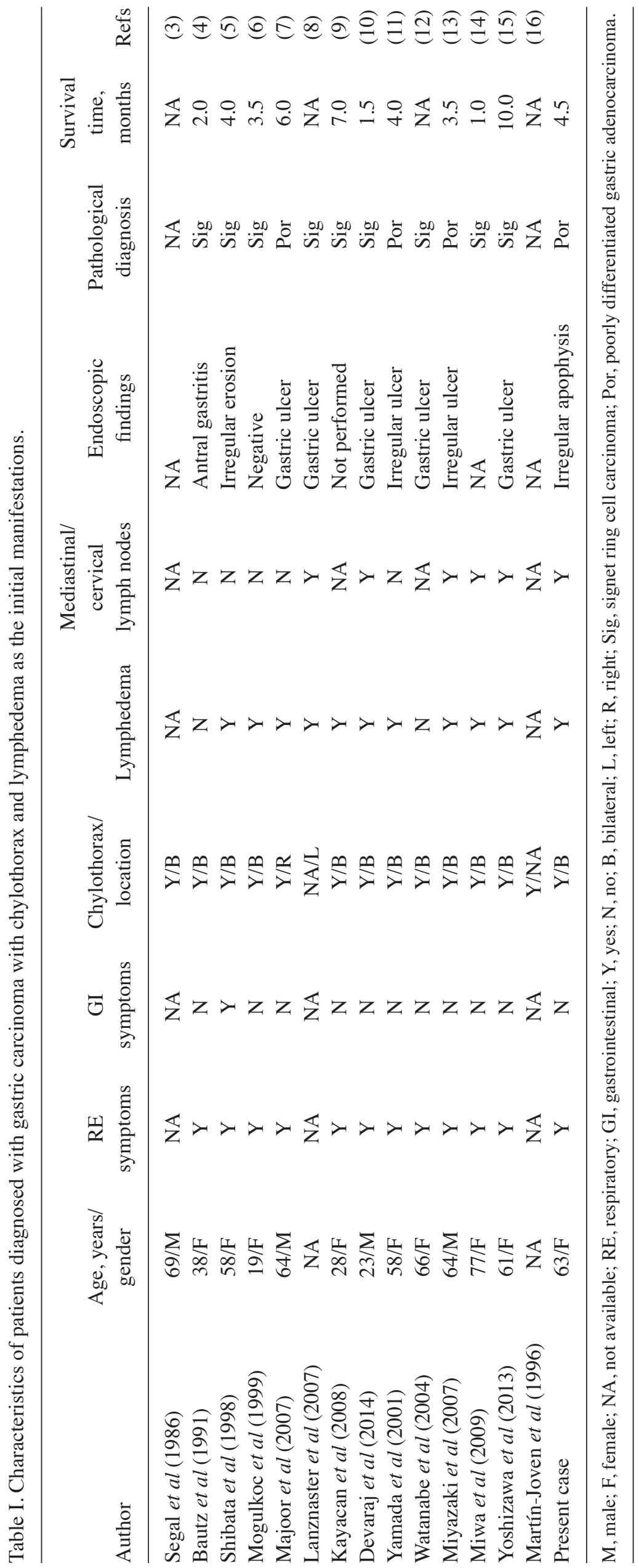


adenocarcinoma $(7,11,13)$. Eleven of these patients presented with bilateral chylothorax with a right effusion predominance. By the time chylothorax was diagnosed, the majority of patients had missed the opportunity for surgery and succumbed a few months later (mean time, 4.3 months).

Limited information on the mechanism of gastric carcinoma-induced chylothorax has been provided in the literature. The thoracic duct is primarily located at the right side of the pleura; this may help explain the development of bilateral chylothorax, particularly the right effusion predominance (17). Dervaraj et al (10) reported the case of an enlarged left supraclavicular lymph node measuring $2.2 \times 1.3 \mathrm{~cm}$, which led to the compression of the left internal jugular vein by $80 \%$ compared with the right internal jugular vein. Dervaraj et al considered that the increase in thoracic duct pressure caused by the compressing enlarged lymph node had an important role in the formation of chylothorax. However, as a large number of collateral and lymphovenous networks exist in the backflow of the thoracic duct $(17,18)$, Shibata et al (5) stated that merely the compression of the thoracic duct was insufficient to cause chylothorax. Yoshizawa et al (15) instead proposed that the invasion of the metastatic mediastinal lymph nodes into the thoracic duct was the cause of chylothorax. However, mediastinal and enlarged supraclavicular lymph nodes were absent in 5/14 cases (4-7,11). Metastatic tumor cells infiltrating the lymph vessels close to the surface of the skin of patients were detected by biopsies in the studies by Bautz et al (4), Shibata et al (5) and Mogulkoc et al (6). Majoor et al (7) suggested that the invasion of metastatic tumor cells into the thoracic duct was the direct cause of chylothorax. In the present case, no obvious mediastinal enlarged lymph nodes were detected by the CT scan; however, an enlarged supraclavicular lymph node was observed. Based on the data collected from the present study and the existing literature, the conclusion was drawn that the invasion of metastatic tumor cells into the thoracic duct was the primary cause of chylothorax, and that the compression of enlarged, metastatic external lymph nodes may also contribute to the occurrence of chylothorax.

Of note, chylothorax was found to be accompanied by lymphedema in 10/14 cases $(5-11,13-15)$. There are two types of lymphedema: Primary lymphedema, which is caused by lymphatic malformation, and secondary lymphedema, which is a result of obstruction or disruption of the lymphatic system, for example, as a consequence of tumors, surgery, trauma, infection and inflammation (19). As no other underlying cause could be identified, lymphedema was considered to be caused by the micrometastasis of gastric carcinoma cells obstructing or infiltrating into the lymphatic networks located close to the skin. The association between chylothorax/lymphedema and gastric carcinoma has not been previously explained. The presentation of lymphedema preceded that of chylothorax 4 and 1.5 years in the reports of Shibata et al (5) and Mogulkoc et al (6), respectively. This phenomenon was also observed in the present case, in which the lymphedema presented 4 months prior to the chylothorax. It has been shown that lymphatic vessels closely intercommunicate through collateral circulation, resulting in lymph reflux into the thoracic duct (17). We propose that the close association between lymphedema/chylothorax and gastric carcinoma in the cases reviewed herein, including the present case, may be a consequence of the infiltration of gastric carcinoma cells into the lymphatic circulatory system. First, the micrometastatic cells arrive at the lymphatic networks located close to the surface of the skin causing lymphedema. Subsequently, the tumor cells spread throughout the lymphatic circulatory system, resulting in chylothorax, or, at an advanced stage, developing into metastatic retroperitoneal lymph nodes.

In conclusion, differential diagnosis of chylothorax of unknown cause should consider gastric carcinoma regardless of gastrointestinal symptoms. Additionly, early detection of malignant tumors should be performed when patients present with lymphedema of uncertain causes. The findings of the present and previous studies suggest that chylothorax and lymphedema in gastric carcinoma may be a result of the infiltration of gastric carcinoma cells into the lymphatic circulatory system.

\section{References}

1. Romero S: Nontraumatic chylothorax: Curr Opin Pulm Med 6: 287-291, 2000.

2. Schild HH, Strassburg CP, Welz A and Kalff J: Treatment options in patients with chylothorax. Dtsch Arztebl Int 110: 819-826, 2013.

3. Segal R, Waron M, Reif R and Zecler E: Chylous ascites and chylothorax as presenting manifestations of stomach carcinoma. Isr J Med Sci 22: 897-899, 1986.

4. Bautz JB, Delaney MD, Mostaghim R and Lodato RF: Chylothorax as presenting manifestation of adenocarcinoma with probable gastric primary. Chest 99: 1044-1045, 1991.

5. Shibata K, Kitagawa S, Fujimura M and Matsuda T: Chylothorax associated with inflammatory carcinoma. Intern Med 37: 538-541, 1998 .

6. Mogulkoc N, Onal B, Okyay N, Günel O and Bayindir U: Chylothorax, chylopericardium and lymphoedema-the presenting features of signet-ring cell carcinoma. Eur Respir J 13: 1489-1491, 1999.

7. Majoor CJ, Aliredjo RP, Dekhuijzen PN, Bulten J and van der Heijden HF: A rare cause of chylothorax and lymph edema. J Thorac Oncol 2: 247-248, 2007.

8. Lanznaster G, Adami M, Crivellaro C, Kluge R, Egarter-Vigl E and Wiedermann CJ: Gastric signet-ring cell carcinoma: Unilateral lower extremity lymphoedema as the presenting feature. ScientificWorldJournal 7: 1189-1192, 2007.

9. Kayacan O, Karnak D, Ayşe Can B, Dizbay Sak S and Beder S: Gastric signet-ring cell adenocarcinoma presenting with left arm deep-vein thrombosis and bilateral chylothorax. Clin Appl Thromb Hemost 14: 476-480, 2008.

10. Devaraj U, Ramachandran P, Correa M and Dsouza GA: Chylothorax in gastric adenocarcinoma: A case report and systematic review of the English literature. Lung India 31: 47-52, 2014.

11. Yamada M, Kudoh S, Hirata K and Yoshikawa J: Bilateral chylothorax as initial manifestation of gastric cancer. Nihon Kokyuki Gakkai Zasshi 39: 343-346, 2001 (In Japanese).

12. Watanabe K, Yamauchi K, Kobayashi K and Takeda H: A case of gastric cancer initially presented by bilateral chylothorax. Nihon Kokyuki Gakkai Zasshi 42: 415-418, 2004 (In Japanese).

13. Miyazaki S, Noda H, Morita T, Joman M, Okada M, Moriyama Y, Suzuki K and Takeuchi T: A case of gastric cancer detected incidentally following to chylothorax, followed by change in the appearance of pleural effusion with cancer progression. Nihon Shokakibyo Gakkai Zasshi 104: 1359-1364, 2007.

14. Miwa M, Kasamatsu N, Shibata M, et al: A case of gastric cancer with bilateral chylothorax. Nihon Kokyuki Gakkai Zasshi, 47: 1115-1119, 2009 (In Japanese).

15. Yoshizawa K, Sasaki Y, Abe Y, Kanno N, Mizumoto N, Yagi M, Yaoita T, Iwano D, Nagino K, Sato T, et al: Chylothorax in a patient with advanced gastric cancer and mediastinal lymph node metastasis causing thoracic duct obstruction. Nihon Shokakibyo Gakkai Zasshi 110: 1943-1949, 2013 (In Japanese).

16. Martín-Joven A, Fernández Ballesteros A, Cervero M, Marco J and Solís J: Chylothorax as presenting form of a gastric adenocarcinoma. Rev Esp Enferm Dig 88: 880-881, 1996 (In Spanish).

17. Skandalakis JE, Skandalakis LJ and Skandalakis PN: Anatomy of the lymphatics. Surg Oncol Clin N Am 16: 1-16, 2007.

18. Schulman A, Fataar S, Dollrymple R and Tidbury I: The lymphographic anatomy of chylothorax. Br J Radiol 51: 420-427, 1978.

19. Murdaca G, Cagnati P, Gulli R, Spanò F, Puppo F, Campisi C and Boccardo F: Current views on diagnostic approach and treatment of lymphedema. Am J Med 125: 134-140, 2012. 